\title{
TRPV1 Induced Apoptosis of Colorectal Cancer Cells by Activating Calcineurin-NFAT2-p53 Signaling Pathway
}

\author{
Nengyi Hou, ${ }^{1}$ Xuelai He, ${ }^{1}$ Yuhui Yang, ${ }^{2}$ Junwen Fu, ${ }^{1}$ Wei Zhang, ${ }^{1}$ Zhiyi Guo, ${ }^{1}$ Yang Hu, \\ Liqin Liang, ${ }^{1}$ Wei Xie, ${ }^{1}$ Haibo Xiong, ${ }^{1}$ Kang Wang $₫{ }^{1},{ }^{1}$ and Minghui Pang $\mathbb{}^{1}$ \\ ${ }^{1}$ Department of Gastrointestinal Surgery, Sichuan Provincial People's Hospital, University of Electronic Science and \\ Technology of China, Chengdu, China \\ ${ }^{2}$ Department of General Surgery, No. 4 Hospital, Zigong City, Sichuan Province, China
}

Correspondence should be addressed to Kang Wang; pangmh2000@163.com and Minghui Pang; pangshattuck@126.com

Received 10 December 2018; Accepted 24 March 2019; Published 30 April 2019

Academic Editor: Wan-Liang Lu

Copyright (C) 2019 Nengyi Hou et al. This is an open access article distributed under the Creative Commons Attribution License, which permits unrestricted use, distribution, and reproduction in any medium, provided the original work is properly cited.

\begin{abstract}
Background/Aims. TRPV1 is a nonselective $\mathrm{Ca}^{2+}$ channel which has recently been observed in many cancers, while its effect on cell proliferation, apoptosis, metabolism, and cancer development in colorectal cancer (CRC) is still unclear. In this study, we hypothesized that TRPV1 is a tumor suppressor in CRC development as well as the underlying mechanism. Methods. Immunohistochemistry assay was applied to detect the expression of TRPV1 protein in CRC tissues. HCT116 cell proliferation and apoptosis were measured by CCK- 8 and flow cytometry, respectively. Cellular $\mathrm{Ca}^{2+}$ concentration was measured by Fluo-4/AMbased flow cytometer. Apoptosis-related proteins were measured by Western blotting. Results. In this study, we found that TRPV1 expression was significantly decreased in CRC tissues, compared with CRC-adjacent tissues and normal tissues, respectively. Then, we found that the TRVP1 agonist capsaicin treatment inhibited CRC growth and induced apoptosis by activating P53. Subsequent mechanistic study revealed that the TRPV1 induced cytosolic $\mathrm{Ca}^{2+}$ influx to regulate cell apoptosis and p53 activation through calcineurin. Conclusions. This study suggests that TRPV1 served as a tumor suppressor in CRC and contributed to the development of novel therapy of CRC.
\end{abstract}

\section{Introduction}

Colorectal cancer (CRC) is one of the common malignant tumors of digestive system and its incidence is increasing year by year and has been reported as the fourth leading cause of cancer associated death worldwide [1]. Currently, research on the pathogenicity and the underlying molecular mechanism of colorectal cancer is still in its infancy [2]. The occurrence of colorectal cancer needs to undergo the process of normal mucosal epithelium to adenoma, dysplasia, carcinoma in situ, colorectal adenocarcinoma, and metastatic carcinoma [3], involved in multiple genes.

Therefore, in-depth research on the genetic and molecular mechanisms related to the occurrence and development of colorectal cancer is an important theoretical basis for the prevention and treatment of colorectal cancer in the future.
Transient receptor potential oxalic acid subtype 1 (TRPV1) is a nonselective cationic ligand gate channel, belonging to the family of transient receptor potential (TRP) ion channels, as it can be activated by capsaicin, also known as vanilloid receptor 1 [4]. TRPV1 was initially regarded as a key sensor for responses to heat and mechanical and chemical stimuli due to its dominance in afferent sensory neurons [5]. TRPV1, meanwhile, has also been linked to the metabolism, longevity, inflammation, and cancer [6]. Yang $\mathrm{Y}$ et al. reported that the low expression of TRPV1 was contributed to melanoma growth via calcineurin-ATF3-p53 pathway [7]. Conversely, the TRPV1 was highly expressed in prostatic cancer, and the lack of TRPV1 inhibited the spread of prostate cancer cells [8]. The above studies showed that the effect of TRPV1 is related to tumor type. Previous reports have shown that TRPV1 is closely related to intestinal diseases such as translocation, irritable bowel syndrome, and 
colitis $[9,10]$. Recently, studies have shown that inhibiting TRPV1 can increase the apoptosis sensitivity of colorectal cancer cells by regulating the ROS-JNK-CHOP pathway [11]. However, the mechanism of TRPV1 inducing apoptosis of colorectal cancer cells remains to be further studied.

$\mathrm{Ca}^{2+}$ is among the major second messengers for connecting membrane receptor activation and downstream signaling transduction, playing an important role in many fundamental physiological processes, including cell excitability, vitality, apoptosis, and transcription [12]. Recent studies have shown that $\mathrm{Ca}^{2+}$ also contributes to some malignant behaviors in tumors, such as proliferation, invasion, migration, and metastasis [13]. The imbalance of intracellular $\mathrm{Ca}^{2+}$ influx is closely related to the hallmarks of various cancers including colorectal cancer $[14,15]$. Given that TRPV1 is a powerful nonselective $\mathrm{Ca}^{2+}$ channel, we hypothesize that TRPV1 can affect the growth of colorectal cancer cells by regulating $\mathrm{Ca}^{2+}$ dependent signaling. Thus, the aim of the present study was to investigate the role of TRPV1 in colorectal cancer progression and provide a deeper understanding of the causal mechanisms of cancer cell proliferation and apoptosis.

\section{Materials and Methods}

2.1. Reagents. Capsaicin (8-methyl-N-vanillyl-trans-6-nonenamide), Pifithrin- $\alpha$ (2-(2-Imino-4, 5, 6, 7-tetrahydrobenzothiazol-3-yl)-1-p-tolylethanone hydrobromide), and FK506 monohydrate (Tacrolimus) were all obtained from Sigma-Aldrich (Merck KGaA, Darmstadt, Germany). Additional reagents employed in the present study were commercially available and of analytical purity.

2.2. Tissue Samples. A cohort of 10 colorectal cancer (CRC) tissue samples, 10 CRC-adjacent tissue samples, and 6 normal subjects for protein detection was obtained from Sichuan Provincial People's Hospital according to the institutional guidelines. All volunteers signed the informed consent. This study was approved by the Ethics Review Board at the University of Electronic Science and Technology of China (Chengdu, China).

2.3. Immunohistochemistry. Immunohistochemical analysis was performed on paraffin-embedded tissues sections. The antigen retrieval was performed by using 3\% hydrogen peroxide at room temperature for $15 \mathrm{~min}$. Subsequently, the sections were incubated with appropriate primary antibody TRPV1 (Cell Signaling Technology, MA, USA) at $4^{\circ} \mathrm{C}$ overnight. Following rewarmed for $30 \mathrm{~min}$ in a $37^{\circ} \mathrm{C}$ incubator, the sections were incubated with appropriate amount of biotinylated goat anti-rabbit IgG for $30 \mathrm{~min}$ at $37^{\circ} \mathrm{C}$. The SABC-POD Kit (Beijing Solarbio Science \& Technology Co., Ltd., Beijing, China) was used for immunohistochemistry of TRPV1 and then counterstained with hematoxylin. PBS was adopted to substitute for primary antibody as negative control group. A total of five visual fields (magnification, $\times 100$ and $\times 400)$ in each section were randomly selected by using a Nikon computer image system (Nikon, Tokyo, Japan) and then assessed for immunoreactive areas using Image-Pro Plus software.

2.4. Cell Viability Assay. The human colorectal cancer cell line HCT116 was obtained from the Shanghai Institutes of Biological Sciences, Chinese Academy of Sciences (Shanghai, China). HCT116 cells were cultured in DMEM medium supplemented with $10 \%$ Fetal Bovine Serum (FBS; Gibco, CA, USA), incubated at $37^{\circ} \mathrm{C}$ in $5 \% \mathrm{CO}_{2}$.

Cell viability was determined by Cell Counting Kit-8 (CCK-8; Dojindo Laboratories, Kumamoto, Japan) assay. In general, approximately $7 \times 10^{3}$ cells per well were seeded in 96-well plates. Following incubation, the original culture medium was removed and $100 \mu \mathrm{l}$ fresh medium was mixed with CCK- 8 at a ratio of 10:1 which was added to each well for $30 \mathrm{~min}$ at $37^{\circ} \mathrm{C}$. The absorbance value was measured at 450 $\mathrm{nm}$ by a microplate reader (Bio-Rad, CA, USA).

2.5. Annexin V/Propidium Iodide (PI) Double-Staining Assay. An Annexin V-fluorescein isothiocyanate- (FITC-) PI Apoptosis Detection Kit (BD Pharmingen; BD Biosciences, Franklin Lakes, NJ, USA) was used to detect apoptosis. HCT116 cells were incubated with Capsicin, Pifithrin- $\alpha$, or FK506 before collection for apoptosis detection. For detecting purpose, cells were collected and resuspended in $100 \mu \mathrm{l}$ binding buffer $\left(1 \times 10^{5}\right.$ cells) with $5 \mu$ l Annexin V-FITC and $5 \mu \mathrm{l}$ PI (BD Biosciences, Franklin Lakes, CA, USA) and incubated at room temperature $\left(20-25^{\circ} \mathrm{C}\right)$ for $15 \mathrm{~min}$ in the dark. Cell apoptosis was detected using a FACSCalibur ${ }^{\mathrm{TM}}$ Flow Cytometer (BD Biosciences) within $1 \mathrm{~h}$.

2.6. Western Blotting Assay. Cells specimens were lysed using RIPA lysis buffer (Boster, Wuhan, China). The equal amounts of total proteins were separated by $10 \%$ SDS-PAGE gel and then transferred onto a PVDF membrane (Millipore, MA, USA). The membranes were blocked with 5\% skim milk powder at room temperature for $1 \mathrm{~h}$ and incubated with primary antibodies diluted in blocking buffer at $4^{\circ} \mathrm{C}$ overnight. Subsequently, the membranes were washed and incubated with the appropriate HRP-conjugated secondary antibodies for $1 \mathrm{~h}$ at room temperature. Protein bands were detected by an ECL chemiluminescence kit (Millipore) according to the manufacturer's instructions. Protein levels were calculated relative to $\beta$-actin. Primary antibodies were used as follows: rabbit anti-NFAT2 (phospho S237) (Abcam, Cambridge, UK, ab183023), rabbit anti-p53 (\#2527), rabbit anti-Bax (\#5023), rabbit anti-Bcl-2 (\#4223), rabbit anti-cleaved-caspase-3 (\#9664), rabbit anti-NFAT2 (\#8032), and rabbit anti- $\beta$-actin (\#4970) were all from cell signaling (Danvers, MA, USA).

2.7. Cellular $\mathrm{Ca}^{2+}$ Concentration Determination. To measure intracellular $\mathrm{Ca}^{2+}$ in colon cancer cells, we used Fluo-4/AM, a cell-permeable fluorescent $\mathrm{Ca}^{2+}$ indicator. Briefly, the HCT116 cells were seeded at a density of $3 \times 10^{4} /$ well in 12 well plates and treated with capsaicin for $24 \mathrm{~h}$. The cells were washed with Hanks Balanced Salt Solutions (HBSS) three times and then stained with $2 \mu \mathrm{M}$ Fluo-4/AM for 

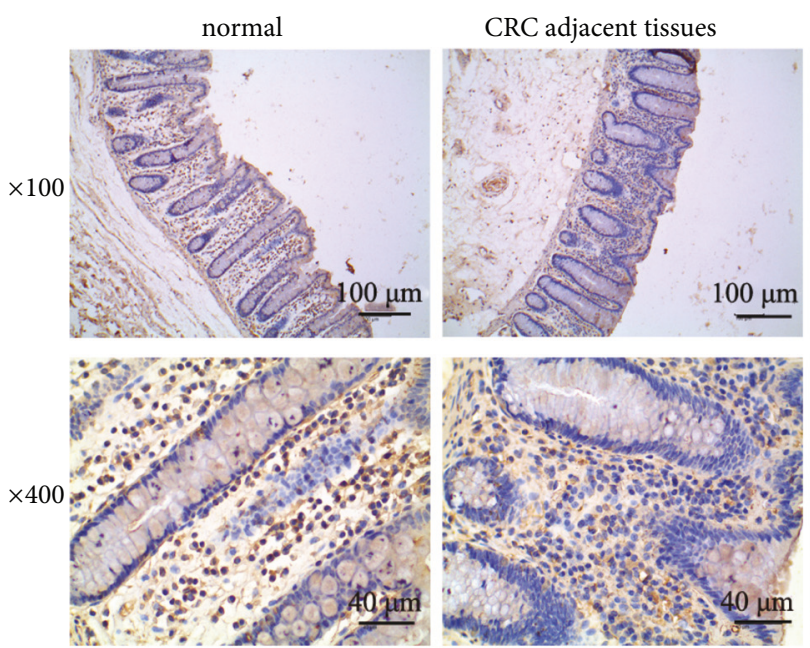

(a)

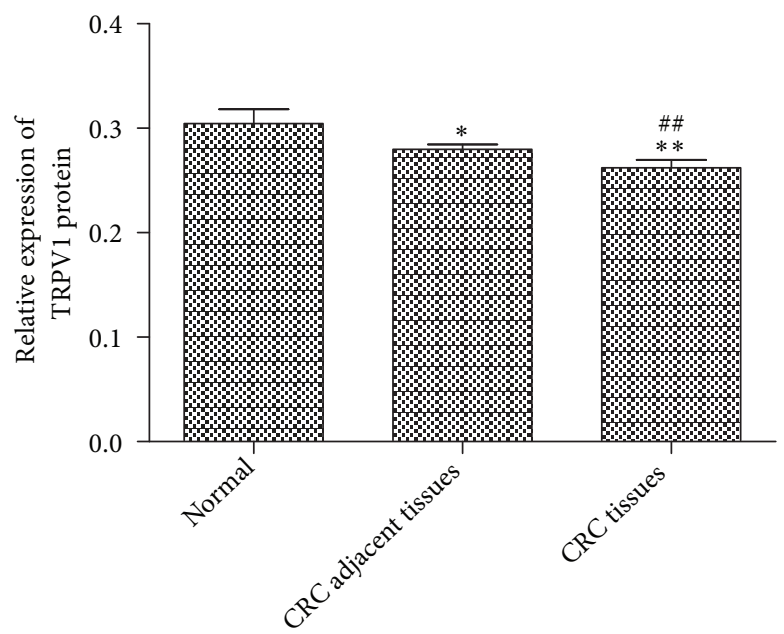

CRC tissues
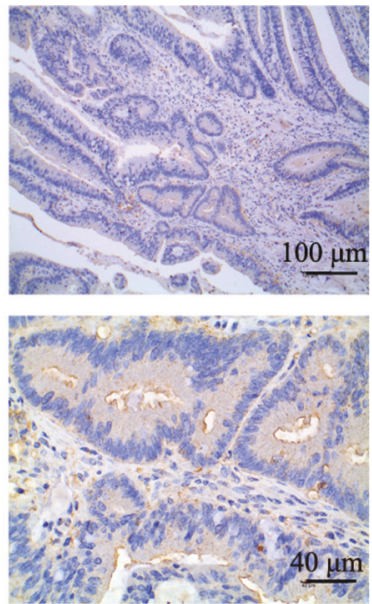

(b)

FIGURE 1: Decrease of TRPV1 expression level in CRC. (a) Immunohistochemical assay was performed to detect the expression of TRPV1 in CRC tissues and adjacent tissues. (b) The expression change of TRPV1 was statistically analyzed (CRC tissues ${ }_{\mathrm{n}=10}$, CRC-adjacent tissues $_{\mathrm{n}=10}$, and normal tissues $\mathrm{n}=10_{10}$. The result was shown as means \pm standard deviation. ${ }^{*} \mathrm{p}<0.05$ and ${ }^{* *} \mathrm{p}<0.01$ versus normal group. ${ }^{\# \#} \mathrm{p}<0.01$ versus CRC-adjacent tissues group.

$30 \mathrm{~min}$ at $37^{\circ} \mathrm{C}$. The results were then evaluated with a flow cytometer. Experiments were repeated at least three times.

\subsection{Immunofluorescence Assay. HCT116 cells were plated} in 12-well plates and incubated overnight for adherence. Subsequently, cells were fixed in $4 \%$ paraformaldehyde for $10 \mathrm{~min}$ at room temperature, permeabilized with $0.1 \%$ Triton X-100, blocked with 5\% BSA, and incubated with primary rabbit anti-NFAT2 antibody (cell signaling, MA, USA) 1 h at room temperature. Slides were washed twice with PBS $/ 0.1 \%$ Tween 20 and incubated with a secondary AlexaFluor 488-conjugated anti-rabbit (green color; Invitrogen, CA, USA) for $1 \mathrm{~h}$ at room temperature. Analyses were performed using ImageJ Software (NIH, Bethesda, MD, USA).
2.9. Statistical Analysis. Statistical analysis was performed using SPSS20.0 software (IBM Corp., Armonk, NY, USA). All data are presented as the mean \pm standard deviation. Differences among multiple groups were compared by one-way analysis of variance (ANOVA) with Dunnett's posttests or two-way ANOVA with Bonferroni's posttests, and differences between two groups were compared by the Dunnett- $t$ test. P $<0.05$ was considered statistically significant.

\section{Results}

3.1. The Expression of TRPV1 Is Decreased in CRC Tissues. To investigate whether TRPV1 is dysregulated in colorectal cancer, we detected the endogenous level of TRPV1 in CRC tissues by using Immunohistochemical assay. As shown in Figure 1, the protein level of TRPV1 was lower in CRC and adjacent tissues, compared with normal tissues. The 
protein level of TRPV1 in CRC tissues was significantly decreased compared with the adjacent tissues. These results demonstrated a significant decrease of TRPV1 expression in CRC, suggesting that TRPV1 may be a tumor suppressor.

\subsection{TRPV1 Induced CRC Cell Proliferation Inhibition and} Apoptosis by Activating p53. To explore the role of TRPV1 in CRC growth, the CRC cell line HCT 116 was treated with capsaicin, a powerful TRPV1 agonist. As a result, capsaicin treatment significantly inhibited HCT116 cell proliferation and induced cell apoptosis, while the proliferation inhibition and apoptosis of HCT 116 cells were significantly decreased following pretreatment with Pifithrin- $\alpha$, a powerful p53 inhibitor, compared with capsaicin group (Figures 2(a) and 2(b)). Furthermore, TRPV1 activation led to prominent upregulation of Bax, cleaved-caspase 3 and p53, and downregulation of Bcl-2, while the apoptosisrelated protein expression was inhibited following pretreatment with Pifithrin- $\alpha$ (Figures 2(c) and 2(d)). These results indicated that the TRPV1 inhibited CRC cell proliferation and induced CRC cell apoptosis through activating p53.

\subsection{TRPV1 Increased Cytosolic $\mathrm{Ca}^{2+}$ Influx and NFAT Protein} Expression Level. We then investigated the mechanism of TRPV1 in regulating HCT116 cell apoptosis. First, we used Fluo-4/AM-based flow cytometer to measure intracellular calcium concentration following TRPV1 activation. As shown in Figure 3(a), the intensity of fluorescence was significantly increased following capsaicin treatment, indicating the upregulation of intracellular $\mathrm{Ca}^{2+}$ concentration. Western blotting analysis showed that p-NFAT2 was significantly downregulated following TRPV1 activation, with the NFAT2 protein expression level increased (Figure 3(b)). In parallel, the immunofluorescence analysis showed obvious increase of NFAT2 (Figure 3(c)).

3.4. TRPV1 Promoted CRC Cell Apoptosis by Activating Calcineurin. We forwardly investigated whether TRPV1 regulated CRC cell apoptosis via calcineurin. The CRC cell line HCT116 was treated with FK506, an inhibitor of calcineurin, following capsaicin treatment. As shown in Figure 4(a), the treatment of FK506 led to decrease of NFAT2 protein compared with calcineurin group, indicating it was functional in suppressing calcineurin activation. Then, flow cytometer results showed that the FK506 significantly reversed the effect of capsaicin on cell apoptosis (Figures 4(b) and 4(c)). In addition, the apoptosis-related protein including Bax, cleaved-caspase 3 and p53 and antiapoptosis protein Bcl-2 was altered correspondingly (Figures 4(d) and $4(\mathrm{e}))$. These results suggested that TRPV1 promoted cell apoptosis and p53 activation through activating calcineurin.

\section{Discussion}

TRPV1 is a ligand-gated $\mathrm{Ca}^{2+}$-permeable ion channel and involved in $\mathrm{Ca}^{2+}$ transport and then maintains the introcellular calcium level [16]. The effects of TRPV1 expression on tumorigenesis and prognosis are different in various types of tumor. The decrease of TRPV1 expression in renal cell carcinoma was significantly associated with tumor Fuhrman grades and histopathological subtypes [17], while the intracellular aggregated TRPV1 was associated with lower survival in breast cancer patients [18]. At present study, we first found that the TRPV1 was lowly expressed in CRC tissues compared with CRC-adjacent tissues and normal subjects, which prompted us to speculate TRPV1 as a tumor suppressor. To clarify the role of TRPV1 in CRC, HCT116 cells were treated with capsaicin, a powerful TRPV1 agonist. As expected, capsaicin treatment markedly inhibited the proliferation of HCT116 cells. Apoptosis is a natural barrier against cancer. Our study showed that the capsaicin treatment significantly induced cell apoptosis and led to prominent upregulation of apoptosis-related proteins. Furthermore, to confirm whether TRPV1 promoted cell apoptosis via p53, we first inhibited p53 expression by Pifithrin- $\alpha$ and then treated with capsaicin. As a result, the proliferation inhibition and apoptosis of HCT116 cells were significantly decreased following pretreatment with Pifithrin- $\alpha$, indicating that the TRPV1, the activation of P53, mediated the proapoptotic role of TRPV1 in CRC.

$\mathrm{Ca}^{2+}$ is known as an essential second messenger that controls various cell physiological functions. Since TRPV1 is a nonselective $\mathrm{Ca}^{2+}$ channel and intracellular $\mathrm{Ca}^{2+}$ homeostasis is critical for the survival and growth of colorectal cancer cells [19], we speculated that $\mathrm{Ca}^{2+}$-dependent effectors were involved in. At present study, we found that the intensity of fluorescence was significantly increased in HCT116 cells following capsaicin treatment, indicating the upregulation of intracellular $\mathrm{Ca}^{2+}$ concentration. Calcineurin is among the most canonical transponders of $\mathrm{Ca}^{2+}$-dependent signal transduction cascade involved in the control of cell cycle progression [20] and cell apoptosis [21] through the dephosphorylation and subsequent nuclear translocation of the downstream transcriptional factor NFAT2 [22]. The $\mathrm{Ca}^{2+} /$ Calcineurin/NFAT signaling plays an important role in cancerogenesis [23] and served as a novel therapeutic target in leukemia and solid tumors [24]. Therefore, we wondered whether calcineurin mediated the effect of TRPV1. Our study showed that the phosphorylated NFAT2 was markedly downregulated following capsaicin treatment, with the NFAT2 protein expression level increased, indicating the activation of calcineurin. We forwardly investigated whether TRPV1 regulated CRC cell apoptosis via calcineurin. FK506, an inhibitor of calcineurin, acts via the complex of FK506 and FK506-binding protein binding to calcineurin to prevent calcineurin-mediated dephosphorylation, commonly used in treating various autoimmune diseases [25]. At present study, we found that the treatment of FK506 led to decrease of NFAT2 protein in HCT116 cells, indicating that it was functional in suppressing calcineurin activation. The flow cytometer results showed that the FK506 significantly reversed the effect of capsaicin on cell apoptosis, and the apoptosis-related proteins were altered correspondingly, indicating that the 


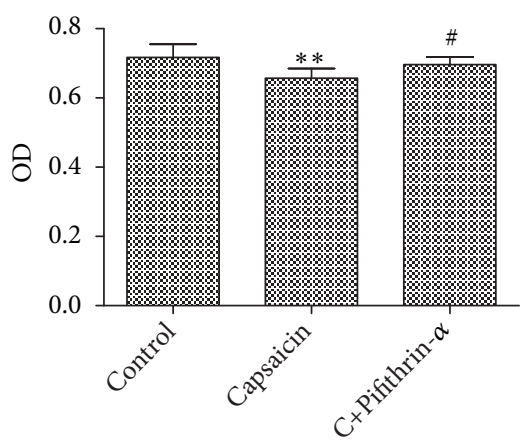

(a)
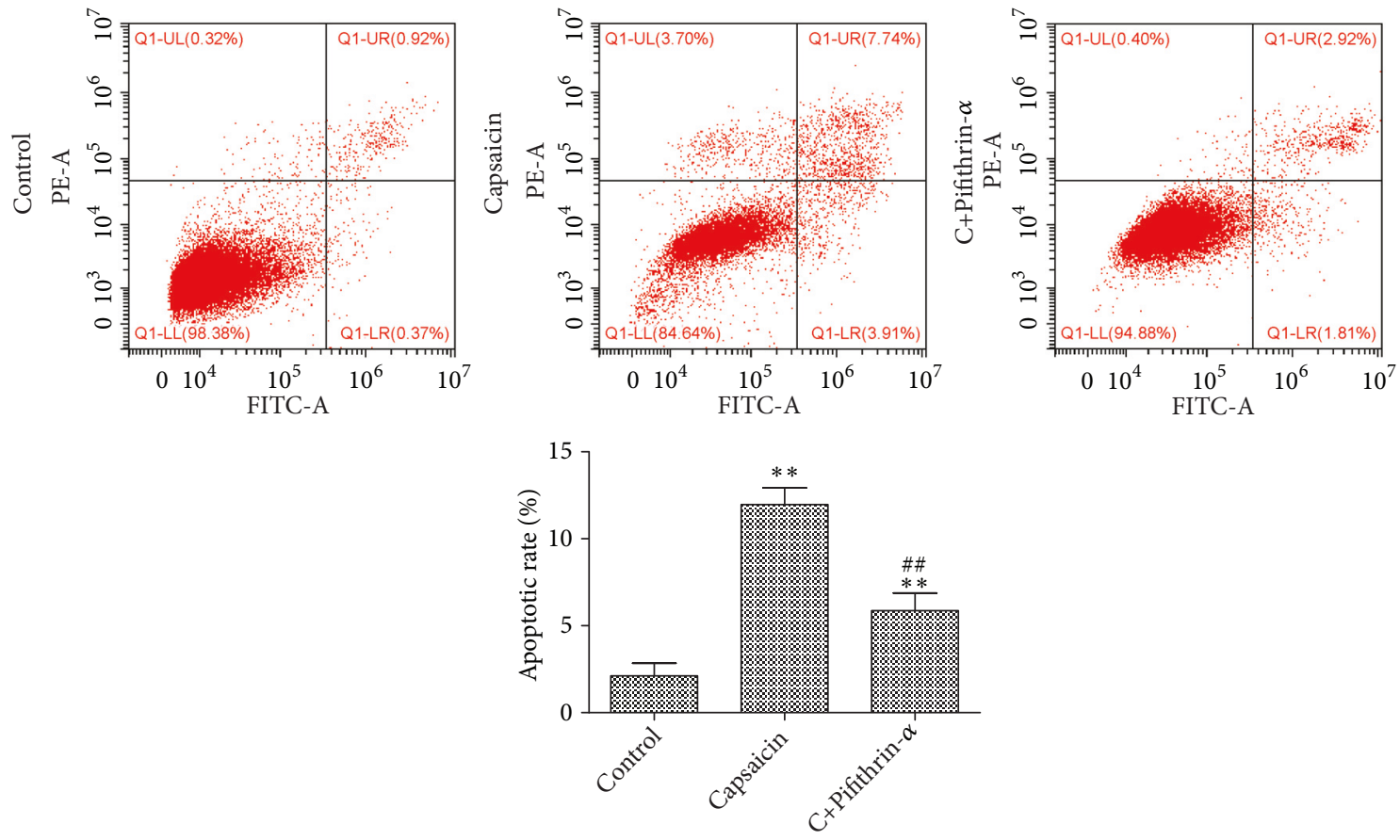

(b)

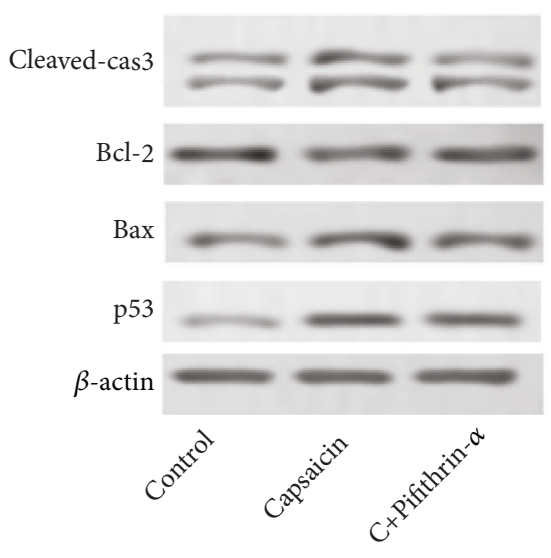

(c)

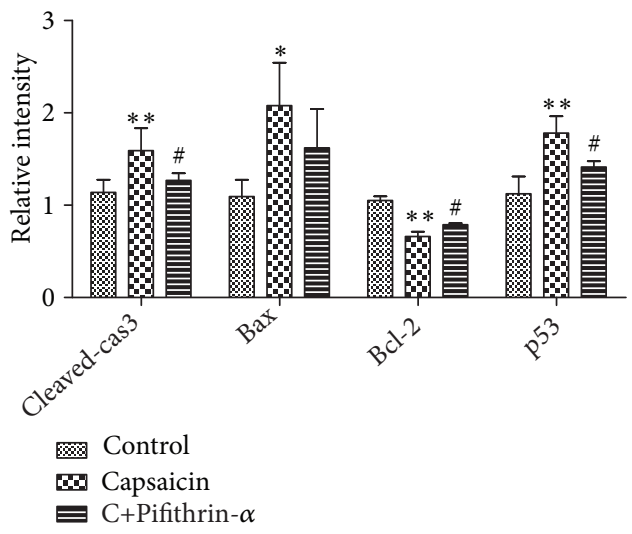

(d)

FIGURE 2: TRPV1 promoted CRC cell apoptosis through activating p53. HCT116 cells were incubated with capsaicin (50 $\mu \mathrm{M})$ in the absence or presence of Pifithrin- $\alpha(20 \mu \mathrm{M})$. (a) Cell viability was determined by CCK- 8 assay following indicated treatment. (b) Cell apoptosis were detected by flow cytometry. (c) The expression levels of apoptosis-related protein were examined by Western blotting. (d) The relative intensity was shown as a bar graph. The result was shown as means \pm standard deviation. ${ }^{*} \mathrm{p}<0.05$ and ${ }^{* *} \mathrm{p}<0.01$ versus control group. ${ }^{\# \#} \mathrm{p}<0.01$ and ${ }^{\#} \mathrm{p}<0.05$ versus capsaicin group. 


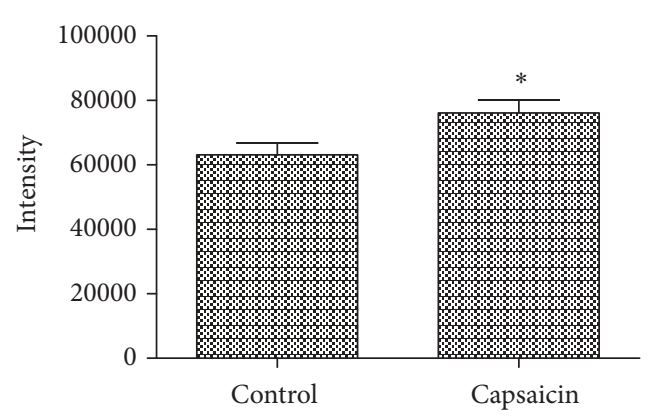

(a)
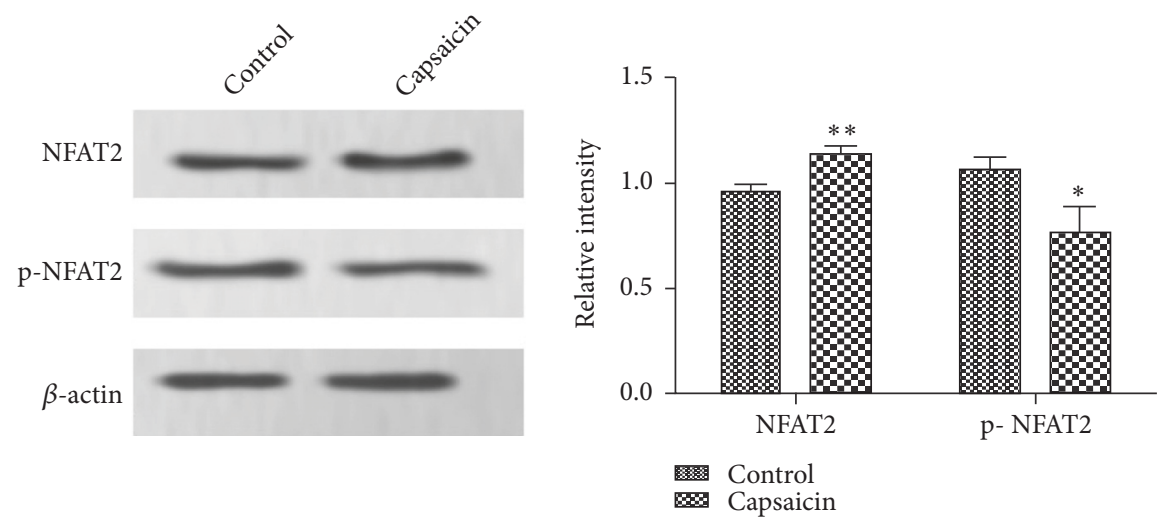

(b)
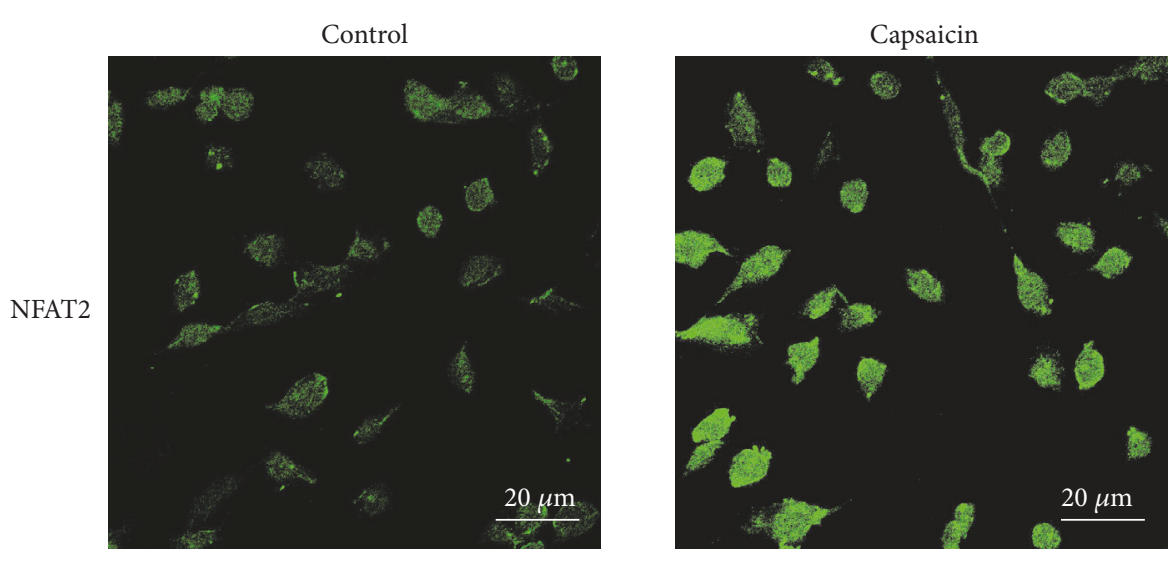

(c)

FIGURE 3: Increased cytosolic $\mathrm{Ca}^{2+}$ influx and NFAT protein induced by TRPV1 overexpression. (a) Measurement of $\mathrm{Ca}^{2+}$ influx by staining with Fluo-4 AM in HCT116 cells following treated with capsaicin $(50 \mu \mathrm{M})$ and then detected by using flow cytometer. (b) The expression levels of p-NFAT2 and NFAT2 protein were examined by Western blotting, and the relative intensity was shown as a bar graph. (c) The expression level of NFAT2 protein was examined by immunofluorescence assay; images were observed by fluorescence microscopy (magnification, $\times 400$ ). The result was shown as means \pm standard deviation. ${ }^{*} \mathrm{p}<0.05$ and ${ }^{* *} \mathrm{p}<0.01$.

TRPV1 promoted cell apoptosis and p53 activation through activating calcineurin.

In summary, we measured the TRPV1 with low expression in CRC tissues and demonstrated that the overexpression of TRPV1 by capsaicin treatment could inhibit cell and increase cell apoptosis in HCT116 cells through activating p53. Moreover, the proapoptotic effect of TRPV1 was attributed to the increased $\mathrm{Ca}^{2+}$ influx and activation of calcineurin. Taken together, we demonstrated TRPV1 as a potent tumor suppressor by activating calcineurin-NFAT2p53 signaling pathway, potentially offering new molecular targets for treatment of CRC.

\section{Data Availability}

The datasets used or analyzed during the current study are available from the corresponding author on reasonable request. 

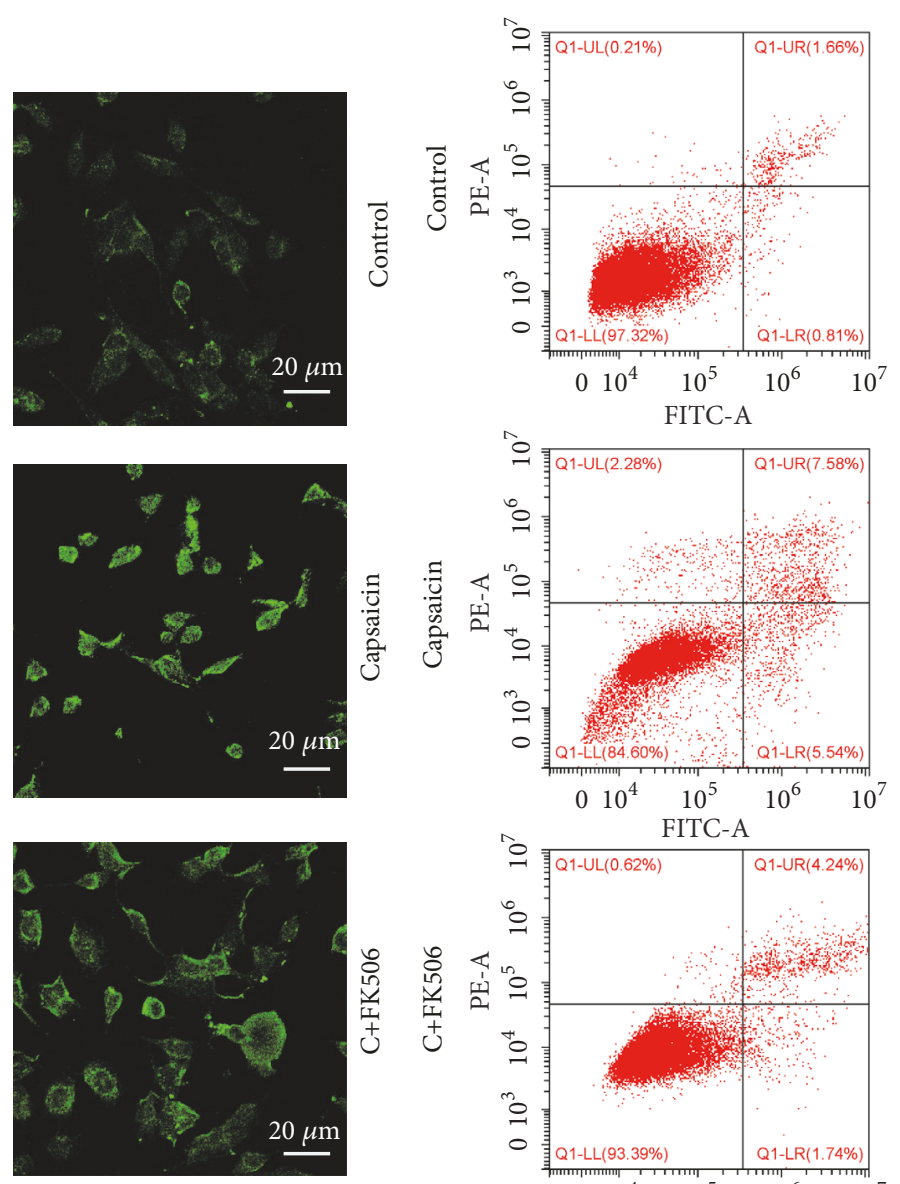

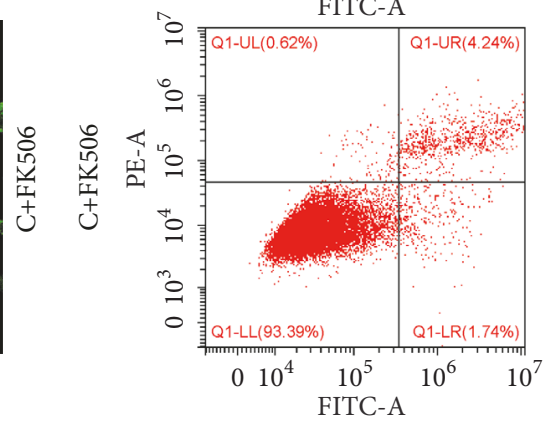

(b)

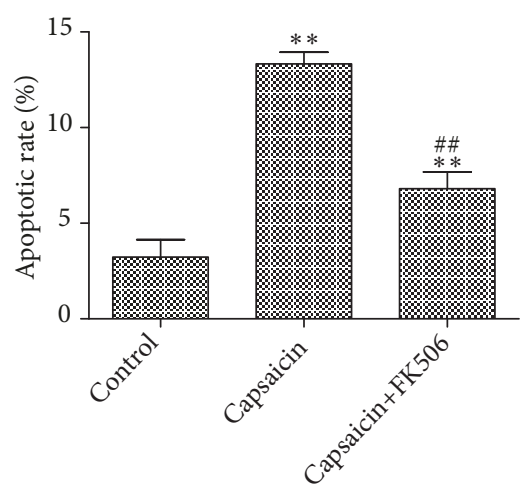

(c)

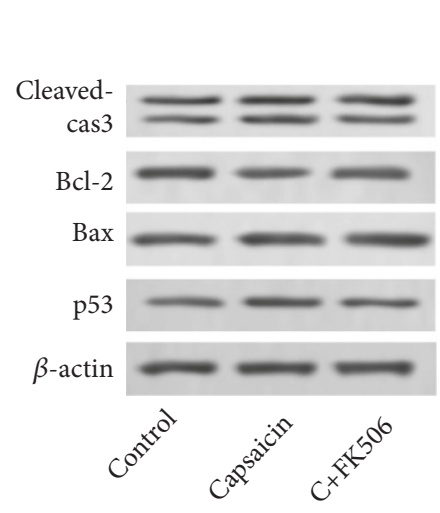

(d)

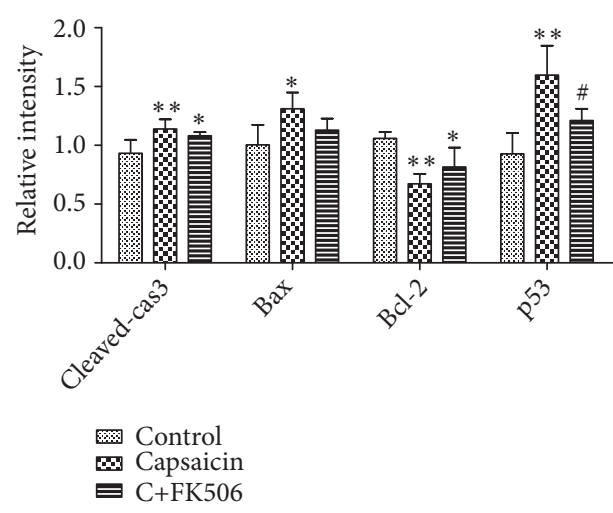

(e)

FIGURE 4: TRPV1 induced cell apoptosis through activating calcineurin. HCT116 cells were incubated with capsaicin $(50 \mu \mathrm{M})$ in the absence or presence of FK506 $(4 \mu \mathrm{M})$. (a) The expression level of NFAT2 protein was examined by immunofluorescence assay; images were observed by fluorescence microscopy (magnification, $\times 400$ ). (b) Cell apoptosis were detected by flow cytometry. (c) The expression levels of apoptosisrelated protein were examined by Western blotting. (d) The relative intensity was shown as a bar graph. The result was shown as means \pm standard deviation. ${ }^{*} \mathrm{p}<0.05$ and ${ }^{* *} \mathrm{p}<0.01$ versus control group. ${ }^{\# \#} \mathrm{p}<0.01$ and ${ }^{\#} \mathrm{p}<0.05$ versus capsaicin group. 


\section{Ethical Approval}

The present study was approved by the Ethics Review Board at the University of Electronic Science and Technology of China.

\section{Consent}

Written informed consent was obtained from all patients.

\section{Conflicts of Interest}

The authors declare that they have no conflicts of interest.

\section{Authors' Contributions}

Nengyi Hou, Xuelai He, and Yuhui Yang contributed equally.

\section{Acknowledgments}

The present study was supported by the Sichuan Provincial Health Department Project (130163) and the Sichuan Provincial Health Department Project (110167).

\section{References}

[1] A. Jemal, F. Bray, M. M. Center, J. Ferlay, E. Ward, and D. Forman, "Global cancer statistics," Ca: A Cancer Journal for Clinicians, vol. 61, no. 2, pp. 69-90, 2011.

[2] W. Wei, Y. Yang, J. Cai et al., "MiR-30a-5p suppresses tumor metastasis of human colorectal cancer by targeting ITGB3," Cellular Physiology \& Biochemistry International Journal of Experimental Cellular Physiology Biochemistry \& Pharmacology, vol. 39, no. 3, article 1165, 2016.

[3] M. Nishikawa, N. Oshitani, T. Matsumoto, T. Nishigami, T. Arakawa, and M. Inoue, "Accumulation of mitochondrial DNA mutation with colorectal carcinogenesis in ulcerative colitis," British Journal of Cancer, vol. 93, no. 3, pp. 331-337, 2005.

[4] S. R. Kim, Y. C. Chung, E. S. Chung et al., "Roles of transient receptor potential vanilloid subtype 1 and cannabinoid type 1 receptors in the brain: neuroprotection versus neurotoxicity," Molecular Neurobiology, vol. 35, no. 3, pp. 245-254, 2007.

[5] W. D. Willis Jr., "The role of TRPV1 receptors in pain evoked by noxious thermal and chemical stimuli," Experimental Brain Research, vol. 196, no. 1, pp. 5-11, 2009.

[6] C. E. Riera, M. O. Huising, P. Follett et al., "TRPV1 pain receptors regulate longevity and metabolism by neuropeptide signaling," Cell, vol. 157, no. 5, pp. 1023-1036, 2014.

[7] Y. Yang, W. Guo, J. Ma, T. Gao, and C. Li, "Downregulated TRPV1 expression contributes to melanoma growth via calcineurin-ATF3-p53 pathway," Journal of Investigative Dermatology, 2018.

[8] M. B. Morelli, C. Amantini, M. Nabissi et al., "Cross-talk between alphalD-adrenoceptors and transient receptor potential vanilloid type 1 triggers prostate cancer cell proliferation," BMC Cancer, vol. 14, no. 1, article 921, 2014.

[9] A. Akbar, Y. Yiangou, P. Facer, J. R. F. Walters, P. Anand, and S. Ghosh, "Increased capsaicin receptor TRPV1-expressing sensory fibres in irritable bowel syndrome and their correlation with abdominal pain," Gut, vol. 57, no. 7, pp. 923-929, 2008.
[10] M. Neri, "Irritable bowel syndrome, inflammatory bowel disease and TRPV1: how to disentangle the bundle," European Journal of Pain, vol. 17, no. 9, pp. 1263-1264, 2013.

[11] B. Sung, S. Prasad, J. Ravindran, V. R. Yadav, and B. B. Aggarwal, "Capsazepine, a TRPV1 antagonist, sensitizes colorectal cancer cells to apoptosis by TRAIL through ROSg-JNK-CHOPmediated upregulation of death receptors," Free Radical Biology \& Medicine, vol. 53, no. 10, pp. 1977-1987, 2012.

[12] M. Zhu, L. Chen, P. Zhao et al., "Store-operated Ca 2+ entry regulates glioma cell migration and invasion via modulation of Pyk2 phosphorylation," Journal of Experimental \& Clinical Cancer Research, vol. 33, no. 1, pp. 1-11, 2014.

[13] N. Prevarskaya, R. Skryma, and Y. Shuba, "Calcium in tumour metastasis: new roles for known actors," Nature Reviews Cancer, vol. 11, no. 8, pp. 609-618, 2011.

[14] Y. T. Kim, S. S. Jo, Y. J. Park et al., "Distinct cellular calcium metabolism in radiation-sensitive RKO human colorectal cancer cells," Korean Journal of Physiology \& Pharmacology Official Journal of the Korean Physiological Society the Korean Society of Pharmacology, vol. 18, no. 6, pp. 509-516, 2014.

[15] M. Gueguinou, D. Crottès, A. Chantôme et al., "The SigmaR1 chaperone drives breast and colorectal cancer cell migration by tuning SK3-dependent Ca2+ homeostasis," Oncogene, vol. 36, no. 25, pp. 3640-3647, 2017.

[16] K. Bley, G. Boorman, B. Mohammad, D. McKenzie, and S. Babbar, "A comprehensive review of the carcinogenic and anticarcinogenic potential of capsaicin," Toxicologic Pathology, vol. 40, no. 6, pp. 847-873, 2012.

[17] Y. Y. Wu, X. Y. Liu, D. X. Zhuo et al., "Decreased expression of TRPV1 in renal cell carcinoma: association with tumor Fuhrman grades and histopathological subtypes," Cancer Management Research, vol. 10, pp. 1647-1655, 2018.

[18] C. Lozano, C. Córdova, I. Marchant et al., "Intracellular aggregated TRPV1 is associated with lower survival in breast cancer patients," Breast Cancer : Targets and Therapy, vol. Volume 10, pp. 161-168, 2018.

[19] M. Gueguinou, D. Crottès, A. Chantôme et al., "The SigmaR1 chaperone drives breast and colorectal cancer cell migration by tuning SK3-dependent $\mathrm{Ca}(2+)$ homeostasis," Oncogene, vol. 36, no. 25, pp. 3640-3647, 2017.

[20] J. Fric, C. X. F. Lim, A. Mertes et al., "Calcium and calcineurinNFAT signaling regulate granulocyte-monocyte progenitor cell cycle via Flt3-L," Stem Cells, vol. 32, no. 12, pp. 3232-3244, 2015.

[21] S. Jayanthi, X. Deng, B. Ladenheim et al., "Calcineurin/NFATinduced up-regulation of the Fas ligand/Fas death pathway is involved in methamphetamine-induced neuronal apoptosis," Proceedings of the National Acadamy of Sciences of the United States of America, vol. 102, no. 3, pp. 868-873, 2005.

[22] M. Dewenter, A. Von Der Lieth, H. A. Katus, and J. Backs, "Calcium signaling and transcriptional regulation in cardiomyocytes," Circulation Research, vol. 121, no. 8, pp. 1000-1020, 2017.

[23] B. Malte and E. Volker, "An emerging role for $\mathrm{Ca}^{2+} / \mathrm{cal}-$ cineurin/NFAT signaling in cancerogenesis," Cell Cycle, vol. 6, no. 1, pp. 16-19, 2007.

[24] M. Hind and G. Jacques, "The calcineurin/NFAT signaling pathway: a novel therapeutic target in leukemia and solid tumors," Cell Cycle, vol. 7, no. 3, pp. 297-303, 2008.

[25] A. Rozkalne, B. T. Hyman, and T. L. Spires-Jones, "Calcineurin inhibition with FK506 ameliorates dendritic spine density deficits in plaque-bearing Alzheimer model mice," Neurobiology of Disease, vol. 41, no. 3, pp. 650-654, 2011. 


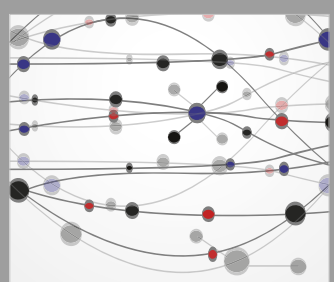

The Scientific World Journal
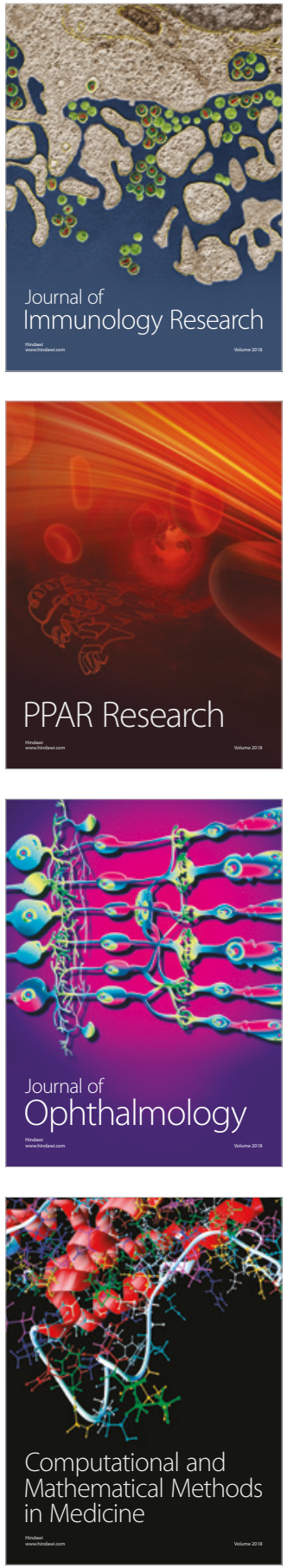

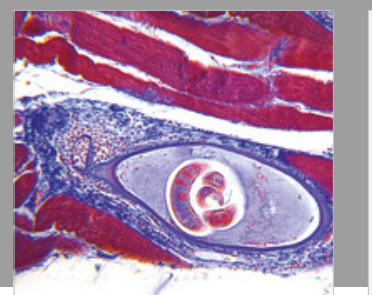

Gastroenterology Research and Practice

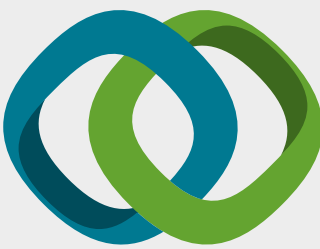

\section{Hindawi}

Submit your manuscripts at

www.hindawi.com
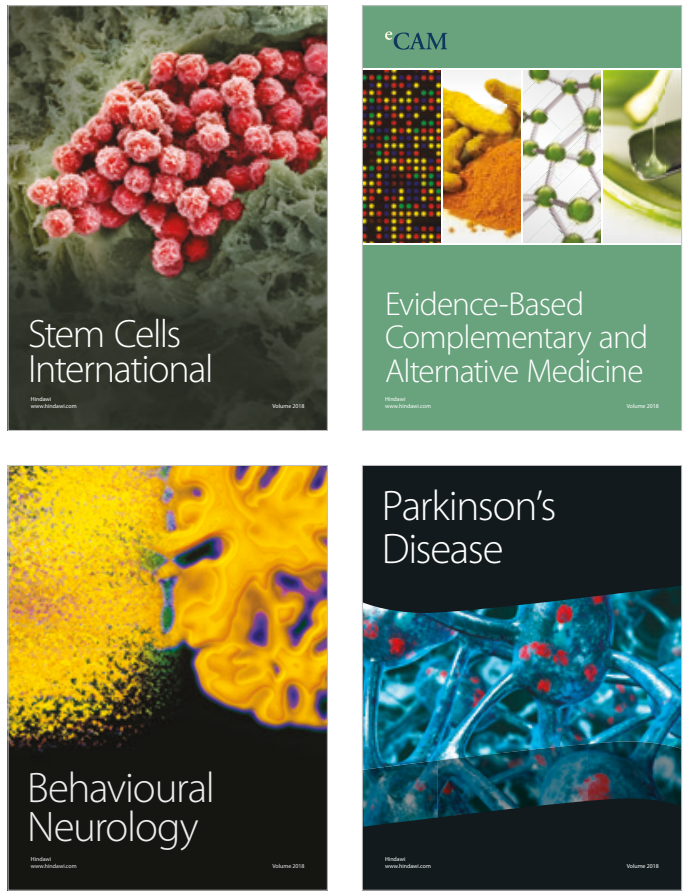

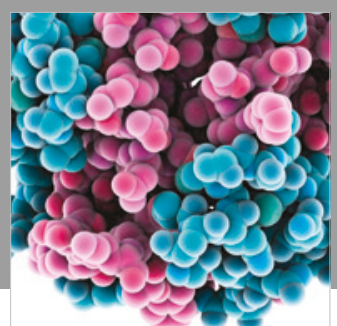

ournal of

Diabetes Research

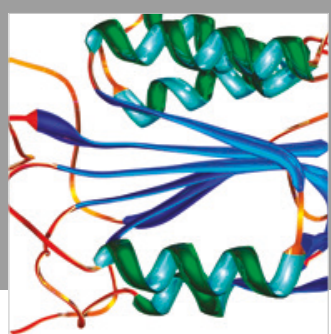

Disease Markers
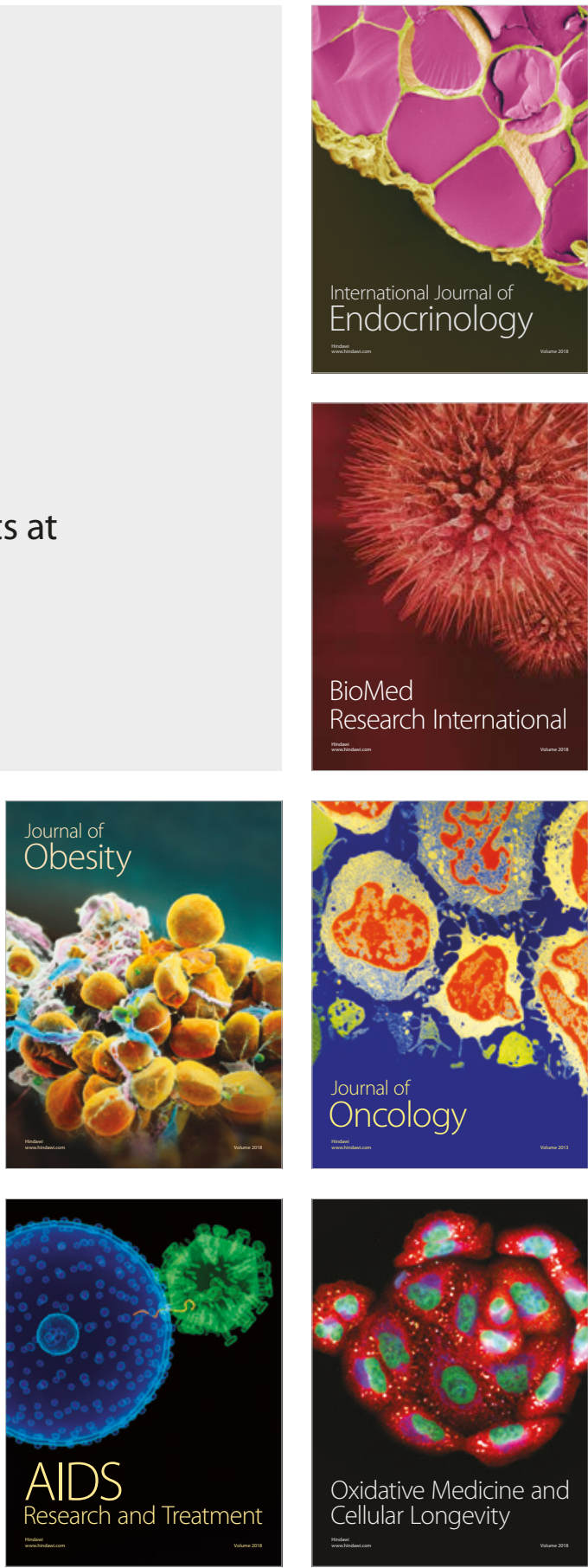